\title{
Interictal mood and personality disorders in temporal lobe epilepsy and juvenile myoclonic epilepsy
}

\author{
G I Perini, C Tosin, C Carraro, G Bernasconi, M P Canevini, R Canger, A Pellegrini, \\ G Testa
}

\begin{abstract}
Background-Mood disorders have been described as the commonest psychiatric disorders in patients with temporal lobe epilepsy. Secondary depression in temporal lobe epilepsy could be interpreted either as an adjustment reaction to $a$ chronic disease or as a limbic dysfunction. To clarify this issue, a controlled study of psychiatric disorders was conducted in different forms of epileptic and non-epileptic chronic conditions.

Methods-Twenty outpatients with temporal lobe epilepsy, 18 outpatients with juvenile myoclonic epilepsy-a primary generalised seizure disorder-20 matched type I diabetic patients, and 20 matched normal controls were assessed by a structured interview (SADS) and by self rating scales (Beck depression inventory (BDI) and the state and trait anxiety scales STAIX1 and STAIX2).
\end{abstract}

Results-Sixteen $(80 \%)$ patients with temporal lobe epilepsy fulfilled the criteria for a psychiatric diagnosis at the SADS interview with a significantly higher frequency than patients with juvenile myoclonic epilepsy $(22 \%)$ and diabetic patients $(10 \%) \quad(P<0.0001)$. The most frequent disorder in temporal lobe epilepsy was a mood disorder: 11 (55\%) patients with temporal lobe epilepsy had depression compared with three patients with juvenile myoclonic epilepsy and two diabetic patients $(P<0.001)$. Eight patients with temporal lobe epilepsy with an affective disorder also had a comorbid personality or anxiety disorder. Patients with temporal lobe epilepsy scored significantly higher on BDI, STAIX1, and STAIX2 than the three control groups $(P<0.001, P<0.01, P<0.001)$.

Conclusions-Patients with temporal lobe epilepsy have a higher incidence of affective and personality disorders, often in comorbidity, than patients with juvenile myoclonic epilepsy and diabetic patients suggesting that these psychiatric disorders are not an adjustment reaction to a chronic disease but rather reflect a limbic dysfunction.

(F Neurol Neurosurg Psychiatry 1996;61:601-605)

Keywords: temporal lobe epilepsy; juvenile myoclonic epilepsy; psychiatric disorders
There is a longstanding debate regarding the relation between interictal psychiatric disorders and partial epilepsy arising from the temporal lobe (temporal lobe epilepsy). In fact, as Stevens noticed ${ }^{1}$ several early reports ${ }^{2-5}$ yielded negative findings when comparing temporal lobe epilepsy with non-temporal lobe epilepsy on self rating scales. However, these studies were not entirely satisfactory from a diagnostic and methodological viewpoint: some misgrouping in the epileptic sample may have occurred, especially some misdiagnosing of patients with temporal lobe epilepsy in the primarily generalised seizures group. Moreover, relying only on self rating scales, especially the Minnesota multiphasic personality inventory (MMPI) for an accurate psychiatric diagnosis is no longer considered a correct methodological procedure. Later studies, some using MMPI, others adopting more updated diagnostic classifications, showed a higher incidence of psychopathology, mainly of depression and anxiety in temporal lobe epilepsy compared with non-temporal lobe epilepsy. ${ }^{6-8}$

Affective disorders such as "endogenous" or major depressive episodes have been described as the commonest psychiatric disorders in patients with epilepsy9-13 and particularly in patients with temporal lobe epilepsy ${ }^{14}$ when compared with normal controls. Rates of suicide attempts have been reported to be four to five times higher than expected in epileptic patients and up to 25 times higher in patients with temporal lobe epilepsy. ${ }^{15-17}$ Depressive symptoms are more frequent in patients with left side onset than those with right or bilateral onset. ${ }^{18-21}$ Bromfield et al, using PET with deoxyglucose, have recently shown that patients with temporal lobe epilepsy with a high depressive score, besides their temporal lobe foci, have a bilateral reduction in inferior frontal glucose metabolism, similar to that in primary depression and in depression secondary to Parkinson's disease, indicating a putative neurobiological substrate for depression secondary to temporal lobe epilepsy. ${ }^{20}$ According to a subsequent study by Victoroff $e t$ $a l^{21}$ in patients with medically intractable temporal lobe epilepsy, both left temporal laterality and the degree of temporal hypometabolism (left and right) might contribute to the risk of interictal depression. Depression, on the other hand, could easily be interpreted as an adjustment reaction to a chronic disease often arising at a young age, with an unpredictable course, requiring frequent monitoring and constant drug treatment, and, at times, difficult to con- 
trol. Social stigma may still be attached to the disease in some social environments. In fact, several studies, in which epileptic patients were compared either with patients with non-epileptic neurological conditions, chronic disabling illnesses, or normal controls, showed a higher incidence of depression and anxiety in the epileptic groups, measured mainly by self rated scales. ${ }^{101322}$

In an attempt to clarify these issues, we decided to conduct a controlled study of psychiatric disorders, assessed both by a structured interview and by self rating scales, in outpatients with temporal lobe epilepsy compared with outpatients with juvenile myoclonic epilepsy, a form of primarily generalised seizure disorder. To assess if interictal depression can be merely attributed to an adjustment reaction to epilepsy, we compared patients with temporal lobe epilepsy and patients with juvenile myoclonic epilepsy to matched patients with type I diabetes, which is a chronic medical disorder arising at a young age and requiring constant medication and monitoring.

\section{Methods}

SUBJECTS

We evaluated 20 patients (six male, 14 female, mean (SD) age $35.2(14)$ ) diagnosed both clinically and by EEG as having temporal lobe epilepsy (group 1), according to the revised classification of epilepsies and epileptic syndromes. ${ }^{23}$ Patients were seen at the epilepsy clinic, Department of Neurology, Padova. We also evaluated 18 patients (two male, 16 female, mean age $27(7 \cdot 6)$ with juvenile myoclonic epilepsy (group 2)) (Janz syndrome) seen at the Epilepsy Regional Center, Department of Neurology, San Paolo Hospital, Milan. As controls we studied 20 patients (six male, 14 female, mean age $38.5(15 \cdot 1)$ ) with type I diabetes (group 3) without ocular, neurological, or cardiovascular complications, seen at the diabetic clinic in Vicenza. These patients were matched to patients with temporal lobe epilepsy on major demographic variables.

We also interviewed 20 healthy controls (group 4) (six male, 14 female) matched to temporal lobe epilepsy for major demographic features.

No significant differences were found among the four groups in sex distribution, mean age, level of education, occupation, and marital status.

All patients and controls gave their informed consent for the study.

\section{Materials and procedures}

Both temporal lobe epilepsy and patients with juvenile myoclonic epilepsy were diagnosed by a neurologist on clinical and EEG grounds according to the Revised Classification of the International League Against Epilepsy. ${ }^{23}$

General characteristics (simple (SPS) and complex partial seizures (CPS)) and EEG characteristics were highly indicative of temporal lobe epilepsy for all patients included in the study. ${ }^{23}$
Patients with juvenile myoclonic epilepsy were diagnosed according to the Revised Classification of Epilepsies and Epileptic Syndromes that defines juvenile myoclonic epilepsy as follows: "juvenile myoclonic epilepsy appears around puberty and is characterised by seizures with bilateral, single, or repetitive, arrhythmic, irregular myoclonic jerks, predominantly in the arms. Jerks may cause some patients to fall suddenly. No disturbance of consciousness is noticeable. Often, there are generalised tonic-clonic seizures and, less often, absence. The seizures usually occur shortly after awakening and are often caused by sleep deprivation. Interictal and ictal EEG have rapid, generalised, often irregular spike waves and polyspike waves; there is no close phase correlation between EEG spikes and jerks. Often the patients are photosensitive. The disorder may be inherited and sex distribution is equal. Response to appropriate drugs is good." 2324

Data on age at onset, type and frequency of seizures, results of EEG and brain imaging studies (CT or MRI when available), and type and dose of medications were recorded (tables 1 and 2).

Patients and controls were interviewed by a psychiatrist using the SADS-PE interview. ${ }^{25} \mathrm{~A}$ period including the past 12 months was covered by the interviewer to assess recent sympthomathology. A psychiatric diagnosis according to research diagnostic criteria of Spitzer and Endicott was than made, when full criteria where met. ${ }^{26}$ For the organic personality disorder the diagnosis was made according to the DSM-III-R criteria, which include a cluster of personality changes such as affective instability, exaggerated aggressiveness, a tendency toward humourless verbosity both in writing and speaking, and hyperreligiosity. ${ }^{27}$

We also gave two self rating scales to the subjects to fill out on the day of the interview. These were the Beck depression inventory $(\mathrm{BDI})^{28}$ and the state and trait anxiety scales (STAIX1 and STAIX2). ${ }^{29}$

Beck depression inventory cut offs for depression are: $0-12=$ normal; $13-20=$ mild; $21-30=$ moderate; $>30=$ severe depression. ${ }^{28}$

The STAIX1 scale measures state anxiety, a transitory emotional state with subjective feelings of tension and apprehension and heightened autonomic nervous system activity whereas STAIX2 measures trait anxiety, a relatively stable individual trait in proneness to anxiety. ${ }^{29}$

These scales have already been used in epileptic patients ${ }^{1418} 19$ showing a good correlation with SADS diagnosis.

\section{STATISTICAL ANALYSIS}

Analysis of variance (ANOVA) followed by post hoc analysis was used for comparing the dependent variables among the four groups. A $\chi^{2}$ test was used to evaluate differences in frequency distribution.

\section{Results}

\section{SADS INTERVIEW}

Sixteen $(80 \%)$ patients with temporal lobe 
Table 1 Psychiatric and neurological variables in temporal lobe epilepsy

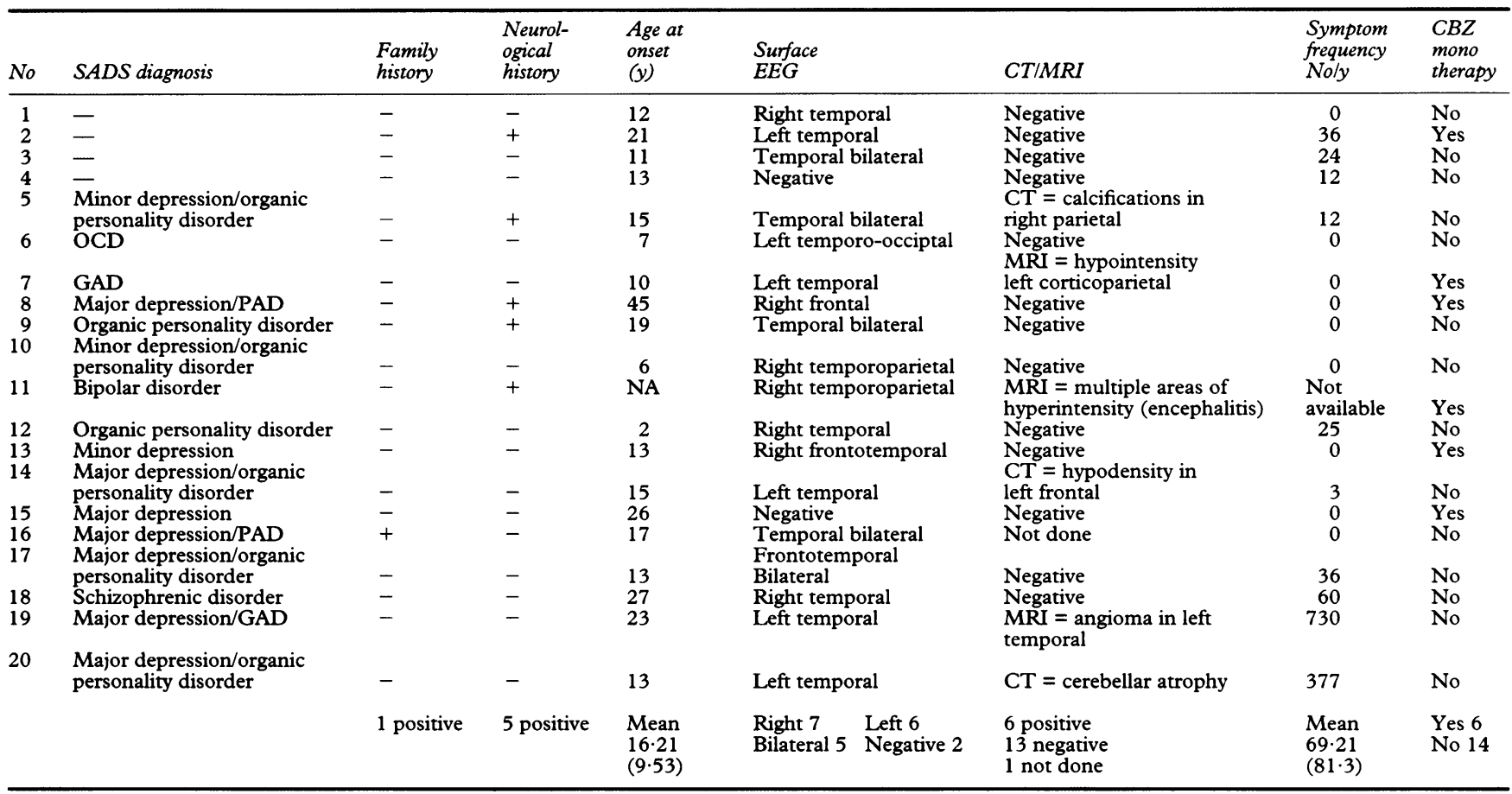

epilepsy fulfilled the criteria for a psychiatric diagnosis at the SADS interview with a significant higher frequency than patients with juvenile myoclonic epilepsy and diabetic patients. In fact only four $(22 \cdot 2 \%)$ patients with juvenile myoclonic epilepsy and two (10\%) diabetic patients met the research diagnostic criteria for a psychiatric disorder $\left(\chi^{2}, \mathrm{P}<0.0001\right.$; tables 1-2).

Eleven (55\%) patients with temporal lobe epilepsy were diagnosed as having a mood disorder: this frequency was significantly higher than in the juvenile myoclonic epilepsy and diabetic groups $\left(\chi^{2}, \mathrm{P}<0.001\right.$; tables $\left.1-2\right)$. The other primary diagnoses in temporal lobe epilepsy were organic personality disorders (two), anxiety disorders (one generalised anxiety disorder (GAD) and one obsessive compulsive disorder (OCD)), and schizophrenic disorder (one).

Comorbidity was present in the temporal lobe epilepsy group: eight patients with temporal lobe epilepsy had an affective diagnosis and also a concomitant psychiatric diagnosis, five of an organic personality disorder and three of an anxiety disorder (table 1).

Of the 24 diagnoses given to the temporal lobe epilepsy group, the most frequent was depression (11 patients, eight unipolar disorder, one bipolar disorder, and two minor depression), followed by organic personality disorder (seven patients), anxiety disorder (five patients: two panic disorders, two generalised anxiety disorders, and one obsessive compulsive disorder), and schizophrenia (one patient).

The diagnoses in juvenile myoclonic epilepsy were three minor depressions and one GAD. None of the four patients with a psychiatric disorder had a comorbid disorder (table 2) Two diabetic patients had a positive diagnosis: one of major depression and PAD, one of atypical depression.

\section{BECK DEPRESSION INVENTORY, STAIX1, AND \\ STAIX 2}

The results on the self rating scales confirmed the higher incidence of psychopathology in the temporal lobe epilepsy group compared with the other groups. The temporal lobe epilepsy group scored significantly higher on BDI (group $1=18 \cdot 1(10.9)$; group $2=8.9(8.4)$; group $3=8.2(7.2)$; group $4=5.0(4.55)$; ANOVA, $\mathrm{P}<0.001$ ), state anxiety (group $1=$ $46 \cdot 7(15 \cdot 8)$; group $2=38 \cdot 4(11 \cdot 7)$; group $3=$ $38.2(10 \cdot 7)$ group $4=34$ (7); ANOVA, $\mathrm{P}<$ 0.01 ), and trait anxiety (group $1=52 \cdot 4(13 \cdot 3)$; group $2=41.4$ (11); group $3=37.9(9 \cdot 6)$; group $4=33.45(7.06) ;$ ANOVA, $\mathrm{P}<0.001)$ compared with the three control groups.

Post hoc analysis showed that the differences were between the temporal lobe epilepsy group and the other three groups, with the exception of a higher score on trait anxiety in juvenile myoclonic epilepsy compared with controls ( $P$ $<0.05$ ).

The BDI, STAIX1, and STAIX2 scores were significantly higher in depressed patients with temporal lobe epilepsy than in nondepressed patients with temporal lobe epilepsy $(\mathrm{BDI}=22.4$ (11) $v 13$ (9) $\mathrm{P}<0.05$; STAIX1 $=53(17) v 39(10), \mathrm{P}<0.05$, STAIX2 $=59$ $(12 \cdot 30 v 44 \cdot 2(9 \cdot 7), \mathrm{P}<0 \cdot 01))$.

\section{SEIZURE RELATED VARIABLES}

We compared the frequency in family history, neurological history, neuroimaging, monotherapy, and the mean age at onset and the seizure frequency in temporal lobe epilepsy and juvenile myoclonic epilepsy (tables 1 and 2). These seizure related variables were selected 
Table 2 Psychiatric and neurological variables in juvenile myoclonic epilepsy

\begin{tabular}{|c|c|c|c|c|c|c|c|c|}
\hline No & SADS diagnosis & $\begin{array}{l}\text { Family } \\
\text { history }\end{array}$ & $\begin{array}{l}\text { Neurol- } \\
\text { ogical } \\
\text { history }\end{array}$ & $\begin{array}{l}\text { Age at } \\
\text { onset } \\
(y)\end{array}$ & $\begin{array}{l}\text { Surface } \\
E E G\end{array}$ & $C T / M R I$ & $\begin{array}{l}\text { Symptom } \\
\text { frequency } \\
(\text { noly) }\end{array}$ & $\begin{array}{l}\text { Valproate } \\
\text { mono } \\
\text { therapy }\end{array}$ \\
\hline 1 & - & - & - & 16 & sw & Not done & 0 & Yes \\
\hline 2 & - & + & - & 17 & SW & Not done & 12 & Yes \\
\hline 3 & - & - & - & 13 & PSW & Not done & 0 & Yes \\
\hline 4 & - & + & - & 13 & Negative & Not done & 365 & Yes \\
\hline 5 & - & + & + & 14 & PSW & Negative & Not available & Yes \\
\hline 6 & - & - & - & 12 & SW and PSW & Not done & 1 . & Yes \\
\hline 7 & - & - & - & 14 & SW and PSW & Negative & 52 & Yes \\
\hline 8 & - & - & - & 16 & SW and PSW & Not done & 0 & Yes \\
\hline 9 & - & - & - & 16 & SW and PSW & Not done & 12 & Yes \\
\hline 10 & - & - & + & 16 & SW and PSW & Negative & 417 & Yes \\
\hline 11 & - & _- & + & 18 & SW and PSW & Not done & 24 & Yes \\
\hline 12 & - & + & - & 21 & SW & Not done & 2 & Yes \\
\hline 13 & - & - & _- & 12 & Atypical SW & Negative & 12 & Yes \\
\hline 14 & - & + & - & 7 & Atypical SW and PSW & Not done & 365 & Yes \\
\hline 15 & Minor depression & + & - & 14 & Atypical SW & Not done & 0 & Yes \\
\hline 16 & Minor depression & + & - & 15 & SW & Not done & 1 & Yes \\
\hline 17 & PAD & - & - & 24 & SW & Not done & 12 & Yes \\
\hline \multirow[t]{2}{*}{18} & GAD & & & 14 & Negative & Not done & 365 & Yes \\
\hline & & 8 positive & 3 positive & $\begin{array}{l}\text { Mean } 15 \cdot 1 \\
\text { (SD 3.7) }\end{array}$ & & & $\begin{array}{l}\text { Mean 96.5 } \\
\text { (SD 161.8) }\end{array}$ & \\
\hline
\end{tabular}

SW = spike waves; $\mathrm{PSW}=$ polyspike waves .

because most of them are reported risk factors in the development of psychopathology in epilepsy. The two groups were not significantly different on mean age at onset, mean seizure frequency, and positive neurological history. Patients with juvenile myoclonic epilepsy reported a significantly higher frequency in family history and in monotherapy than patients with temporal lobe epilepsy, as expected (respectively $44 \%$ v 5\%, P < 0.005; $100 \%$ v $30 \%, \mathrm{P}<0.0001)$.

Six patients with temporal lobe epilepsy had positive neuroimaging (cerebral CT or MRI). These data were not available in most patients with juvenile myoclonic epilepsy. Only four patients with juvenile myoclonic epilepsy underwent a brain imaging procedure and all were negative (tables 1 and 2).

The two groups were therefore comparable on most seizure variables, with the exception of family history and type of treatment.

\section{Discussion}

Our results clearly indicate that patients with temporal lobe epilepsy have a higher incidence of psychiatric disorders than patients with primary generalised seizures and patients with a chronic disease requiring constant medication and monitoring such as type I diabetes.

Our study lends support to the hypothesis that patients with seizures arising from temporal lobe structures can be more prone to psychopathological dysfunction than patients with primarily generalised seizures. This hypothesis has been sustained by many authors ${ }^{6-817}$ but rejected by others. ${ }^{1}$ However, our study is the first in which both well defined criteria for the diagnosis of seizures according to the international classification of epileptic seizures ${ }^{21}$ and well defined, standardised diagnostic criteria for psychiatric disorders according to the research diagnostic criteria and DSM-III-R classification have been employed. Previous studies, which yielded negative results, may have been biased by misgrouping of epileptic patients (patients with secondarily generalised seizures in the group of primarily generalised seizures). Moreover, all the negative studies relied only on self rating scales for the psychiatric diagnosis.
This procedure is no longer acceptable in psychiatric research. In fact, the use of standardised psychiatric interviews and updated diagnostic criteria, even if often advocated, has been very rarely used in research on the psychiatric manifestations of seizure disorders. ${ }^{11} 1417$

When we considered the diagnostic profile of patients with temporal lobe epilepsy, we found that $55 \%$ of patients received a diagnosis of depression and $35 \%$ of an organic personality syndrome. Comorbidity was high in patients with temporal lobe epilepsy and the most common cluster was an association between depression and organic personality syndrome. Interictal depression has already been reported as an important concomitant of temporal lobe epilepsy in many studies that employed clinical assessment, ${ }^{93031}$ self rating scales, ${ }^{1013}$ or structured interviews. ${ }^{114}$ The present study confirms such an association in patients with temporal lobe epilepsy, but not in patients with primarily generalised seizure disorder.

Based on our results, we can state that having one or more epileptogenic foci in the temporal structures is a risk factor for the development of affective symptoms, whereas having recurrent generalised seizures is not. The neurobiological mechanisms that subserve such an association still need to be clarified. Lateralisation to the left temporal lobe has been found to be more often associated with depression than right or bilateral localisation of the epileptogenic foci in patients with temporal lobe epilepsy. ${ }^{18-20}$ Inferior frontal hypometabolism on 2FDG-PET has been reported in depressed compared with non-depressed patients with temporal lobe epilepsy and this pattern of cerebral metabolism is similar to that present in other primary or secondary types of depression. ${ }^{20}$ Besides the localisation, the degree of temporal hypometabolism has also been correlated with the risk of interictal depression. ${ }^{21}$ However, the role of the limbic system both in primary and secondary depression cannot be thoroughly assessed because a higher resolution would be needed to investigate smaller areas of potential relevance.

In our study, juvenile myoclonic epilepsy was not significantly associated with any psychiatric diagnosis. There are no previous controlled 
studies on psychiatric profile in juvenile myoclonic epilepsy, but only descriptive reports of personality changes. Our only significant finding was a higher trait anxiety on STAIX1, a self report scale. Trait anxiety indicates an apprehensive person in whom anxiety is more a personality characteristic than a temporary status. ${ }^{29}$ We can speculate that the development of such an apprehensive and worried personality could be reinforced by the frequent sudden loss of control on movement and on consciousness in juvenile myoclonic epilepsy. Trait anxiety is high also in temporal lobe epilepsy, as shown by this and by previous studies, ${ }^{14}$ but in this group, state anxiety and depression scores are also increased in the context of an affective disorder. In fact depressed patients with temporal lobe epilepsy scored significantly higher than nondepressed patients on the Beck and state and trait anxiety tests.

Comorbidity of psychiatric diagnosis is another important aspect which emerged in our patients with temporal lobe epilepsy. In fact, comorbidity was present almost exclusively in patients with temporal lobe epilepsy and it was mainly characterised by the association of depression with an organic personality disorder. Organic personality disorder has been described as a common psychiatric complication of temporal lobe epilepsy, which usually arises a few years after the onset of the seizures. ${ }^{32}{ }^{33}$ It is a well characterised behavioural syndrome that has been conceptualised as the opposite of a Kluver-Bucy syndrome, a temporal disconnection syndrome. ${ }^{32} 33$ The temporal "hyperconnection" syndrome usually manifests itself with three major clusters of changes, the circumstantiality, the deepened emotionality, and the hyposexuality. So far the possibility of a frequent association between interictal episodes of depression and personality changes was not considered. In these studies depression was only considered as one symptom related to the increased emotionality and not as a full syndrome. This is the first report that clearly shows a highly frequent comorbidity between affective disorders and personality disorders in patients with temporal lobe epilepsy.

A third, nevertheless important, finding is that patients with temporal lobe epilepsy had a more severe psychopathological profile than patients with a medical chronic disease such as type I diabetes. Patients with diabetes were similar to patients with juvenile myoclonic epilepsy and normal subjects when compared both on SADS and a self rating scale. On the contrary, patients with temporal lobe epilepsy had a higher number of psychiatric diagnoses and higher scores on depression and anxiety scales than diabetic patients. Such differences clearly indicate that interictal psychiatric symptoms and, in particular, interictal affective symptoms, are not simply an adjustment reaction to a chronic medical condition, requiring constant medication and monitoring, but are likely to be secondary to a limbic dysfunction. ${ }^{11}{ }^{17-20}$

In conclusion, temporal lobe epilepsy is a neurological condition with a high proportion of interictal psychiatric manifestations, mainly clinical depression, which are more likely to be related to a limbic dysfunction rather than simply reflect a psychological adjustment to a chronic epileptic or medical condition.

1 Stevens JR. Risk factors for psychopathology in individuals with epilepsy. Adv Biol Psychiatr 1982;8:56-80.

2 Matthews CG, Klove H. MMPI performances in majo motor, psychomotor, and mixed seizure classifications of known and unknown etiology. Epilepsia 1968;11:345-59.

3 Mignone RJ, Donnelly EF, Sadowsky D. Psychological and neurological comparisons of psychomotor and non-psychomotor epileptic patients. Epilepsia 1970;11:345-59.

4 Glass DH, Mattson RH. Psychopathology and emotional precipitation of seizure in temporal lobe epileptics. Proceedings of the 81st Annual Convention of the American Proceedings of the 81st Annual Convention
Psychological Association 1973;8:425.

5 Standage KF, Fenton GW. Psychiatric symptom profiles of patients with epilepsy: a controlled investigation. Psychol Med 1975;5:152-60.

6 Rodin EA, Katz M, Lennox K. Differences between patients with temporal lobe seizures and those with other form of epileptic attacks. Epilepsia 1976;17:313-20.

7 Shukla GD, Srivastava ON, Katiyar BC, Joshi V, Mohan PK. Psychiatric manifestations in temporal lobe epilepsy: a controlled study. Br F Psychiatry 1979;135:411-7.

8 Dikmen S, Hermann BP, Wilensky AJ, Rainwater G Validity of the Minnesota multiphasic personality inventory (MMPI) to psychopathology in patients with epilepsy. f Nerv Ment Dis 1983;171:1 14-22.

9 Betts TA. A follow-up study of a cohort of patients with epilepsy admitted to psychiatric care in an English city.

10 Kogeorgos J, Fonagy P, Scott DF. Psychiatric symptom patterns of chronic epileptics attending a neurological clinic: controlled investigation. Br f Psychiatry 1982;140:236-43.

11 Perez MM, Trimble MR. Epileptic psychosis-diagnostic comparison with process schizophrenia. Br $\mathcal{f}$ Psychiatry 1980;137:245-9.

12 Robertson M, Trimble MR, Towsend HRA. Phenomenology of depression in epilepsy. Epilepsia 1987;28:364-72.

13 Mendez MF, Cummings JL, Benson DF. Depression in epilepsy. Significance and phenomenology. Arch Neurol 1986;43:766-70

14 Perini GI. Research Diagnostic Criteria (RDC) mental disorder and self-reported symptoms in outpatients with complex partial seizures (CPS). International fournal of Psychiatry in Medicine 1987;17.

15 Hawton J, Marsack P. Association between epilepsy and attempted suicide. $\mathcal{f}$ Neurol Neurosurg Psychiatry 1980; 43:168-70.

16 Barclough BM. Suicide and epilepsy. In: Reynolds EH, Trimble MR, eds. Epilepsy and psychiatry. New York: Churchill Livingstone, 1981:72-6.

17 Post RM, Uhde TW, Joffe RT, Bierer L. Psychiatric manifestation and implications of seizure disorders. Medical Mimics of Psychiatric Disorders 1986:35-91.

18 Perini GI, Mendius R. Depression and anxiety in complex partial seizures. $\mathcal{f}$ Nerv Ment Dis 1984;172:287-90.

19 Altshuler LL, Devinsky O, Post RM, Theodore W. Depression, anxiety, and temporal lobe epilepsy. Arch Neurol 1990;47:284-8.

20 Bromfield EB, Altshuler L, Leiderman DB, et al. Cerebral metabolism and depression in patients with complex parmetabolism and depression in patients with
tial seizures. Arch Neurol 1992;49:617-23.

21 Victoroff JI, Benson F, Grafton ST, Engel J Jr, Mazziotta JC, Depression in complex partial seizures. Electroencephalography and celebral metabolic correlates. Arch

22 Robertson MM, Channon S, Baker J. Depressive symptomatology in a general hospital sample af outpatients with temporal lobe epilepsy: a controlled study. Epilepsia 1994 35:771-7.

23 Commission on Classification and Terminology of the International League Against Epilepsy. Proposal for revised classification and terminology of epilepsies and epileptic syndromes. Epilepsia 1989;30:389-99.

24 Canevini MP, Mai R, Di Marco C, et al. Juvenile myoclonic epilepsy of Janz: clinical observations in 60 patients. Seizure 1992;1:291-8.

25 Endicott J, Spitzer R. A diagnostic interview: the schedule for affective disorders and schizophrenia. Arch Gen for affective disorders and

26 Spitzer R, Endicott J, Robins E. Research diagnostic criteria: rationale and reliability. Arch Gen Psychiatry 1978;35: 773-82.

27 American Psychiatric Association. Diagnostic and statistical manual of mental disorders (DSM-III-R). Washington: APA, 1987.

28 Beck A, Beamespefer A. Assessment of depression: the depression inventory. Pharmacopsychiatry 1974;7:69-171.

29 Spielberger CD. STAI manual, Palo Alto: Consulting Psychologist Press, 1970.

30 Dominian J, Serafetinides EA, Dewhurst M. A follow-up study of late-onset epilepsy. II. Psychiatric and social findings. $B M \mathcal{F} 1963 ; 5328: 421-35$.

31 Currie S, Heathfield KWG, Henson RA, et al. Clinical course and prognosis of temporal lobe epilepsy. Brain 1971;94:173-90.

32 Blumer D, Benson DF. Psychiatric manifestation of epilepsy. New York: Grune and Stratton, 1982.

33 Bear D, Fedio P. Quantitative analysis of interictal behavior in temporal lobe epilepsy. Arch Neurol 1977;34:454-67. 